\title{
Off-Label-Use und Zulassung: ein Spagat bei der Behandlung von Minderjährigen
}

Die Kinderheilkunde genießt in der Bevölkerung ein hohes Ansehen. Im Gegensatz zu diesem positiven Bild gelten Kinderarzneimittel bei der Pharmaindustrie als „schwieriger Markt“. Aus verschiedenen Gründen streben Firmen oft keine NachZulassung von bei Erwachsenen seit Jahrzehnten zugelassenen Medikamenten an. Daher müssen diese Präparate bei Kindern häufig im Off-label use eingesetzt werden. Besonders dramatisch ist die Situation bei der Behandlung von Neugeborenen und Frühgeborenen. In diesem Bereich werden oft weit mehr als die Hälfte der Medikamente „off label“ verwendet.

Der „Best Pharmaceuticals for Children Act" von 2002 und die EU-Arzneimittelverordnung von 2007 sollten für neu zugelassene Medikamente eine Verbesserung bewirken; die Analysen der bisherigen Effekte dieser Verordnungen waren jedoch zum Teil sehr ernüchternd.

Aber auch die für Kinder und Neugeborene zugelassenen Medikamente sind oft unzureichend untersucht, insbesondere in Bezug auf ihre Nebenwirkungen. Im Bereich der Neuentwicklung von speziellen Medikamenten für Kinder werden, abgesehen von Impfstoffen und extrem hochpreisigen Präparaten für seltene genetische Erkrankungen, kaum neue Produkte erforscht.

Am Beispiel einer großen Kohortenstudie von sehr kleinen Frühgeborenen lässt sich zeigen, dass die Erforschung von Arzneimitteln für Kinder auch positive Auswirkungen auf die Erwachsenenmedizin haben kann. Hierzu trägt folgendes bei:

1. Genetische Identität von Erwachsenen und Kindern: Die in den aktuellen Erwachsenenstudien publizierten genetischen Risikofaktoren für Erkrankungen und bestimmte Eigenschaften lassen sich oft direkt auf Frühgeborene übertragen. So ist ein genetischer Risikoscore für die chronisch obstruktive Lungenerkrankung (COPD) bei Erwachsenen auch bei Frühgeborenen im Alter von 5 Jahren mit einer erniedrigten FEV1 in der Spirometrie assoziiert. Ge- netische Varianten im APOE-Gen, die bei Erwachsenen ein Risikofaktor für Hirnblutungen und die Alzheimer-Erkrankung sind, treten auch bei Frühgeborenen mit Hirnblutungen gehäuft auf.

Kinder sind also in viel höherem Maße „kleine Erwachsene“, als wir bisher glaubten. Aus diesem Grund sind die in Medikamentenstudien bei Früh- und Neugeborenen gewonnenen Daten mit hoher Wahrscheinlichkeit auch sehr gut auf Erwachsene mit vergleichbaren genetischen Risikofaktoren übertragbar.

2. Kinder mit einzigartigen Risikoprofilen: Akute Erkrankungen treten bei Neugeborenen und Frühgeborenen oft in einem sehr engen Zeitfenster auf. Hirnblutungen sind eine häufige Komplikation bei Frühgeborenen; diese Blutungen treten aber praktisch immer innerhalb der ersten 72 Lebensstunden auf. Ein vergleichbar enges Zeitfenster für Hirnblutungen bei Erwachsenen (die den gleichen genetischen Risikofaktoren unterworfen sind) existiert nicht. Die Untersuchung von prophylaktisch gegen Hirnblutungen wirksamen Medikamenten wäre also bei Frühgeborenen viel leichter möglich als bei Erwachsenen.

3. Neue Möglichkeiten bei der Nutzung von „Real-World Data“ in Verbindung mit standardisierten Follow-up-Untersuchungen: Eine hochgradig standardisierte langfristig angelegte Nachuntersuchung von Kindern ist geeignet zur systematischen Untersuchung von Medikamentenwirkungen und Nebenwirkungen. Für das Medikament Dexamethason sind bei Frühgeborenen ungünstige Wirkungen auf die langfristige neurokognitive Entwicklung in randomisierten Studien gut untersucht. Es lässt sich zeigen, dass sich dieser Zusammenhang auch durch eine standardisierte Kohortenstudie mit ausreichend hoher Fallzahl nachweisen lässt.

Expositionsdaten von Kindern im stationären Bereich fallen heute in aller Regel ohnehin als elektronische Verordnungsdaten an. Kombiniert man diese Daten mit einer standardisierten Nachuntersuchung im Alter von 2 oder 5 Jahren sind wesentliche Probleme einer wirksamen Pharmakovigilanz bei Neu- und Frühgeborenen gelöst. Die Umsetzung eines solchen Programms im großen Maßstab ist allerdings nicht einfach. Die so erhobenen Daten können auch für die Medikamentenentwicklung im Erwachsenenbereich hilfreich sein, insbesondere wenn sie mit genetischen Markern kombiniert werden.

Es ist absehbar, dass Frühgeborene, Neugeborene und Kinder auch in Zukunft in wirklich großer Zahl mit nur unzureichend erforschten Medikamenten und Medikamentenkombinationen behandelt werden - ganz unabhängig vom Zulassungsstatus dieser Medikamente. Bei bestimmten Patientengruppen sollten sowohl Medikamenten-Expositionen als auch LangzeitOutcome Daten systematisch erhoben werden. Insbesondere in Kombination mit genetischen Daten lässt sich so die Medikamentensicherheit für Neugeborene und Kinder in weit höherem Maße verbessern als durch vereinzelte Zulassungen von bisher nur off-label verwendeten Medikamenten.

\section{Interessenkonflikte}

Der Autor hat Vortragshonorare von Chiesi und Beraterhonorare von Chiesi und Shire erhalten.

\section{Autor}



Wolfgang Göpel Universitätsklinikum Schleswig-Holstein, Campus Lübeck, Klinik für Kinder- u. Jugendmedizin

Korrespondenzadresse

Prof. Dr. med. Wolfgang Göpel

Universitätsklinikum Schleswig-Holstein Campus Lübeck

Klinik für Kinder- u. Jugendmedizin

Ratzeburger Allee 160

23538 Lübeck

wolfgang.goepel@uksh.de 